\title{
Hairy Cell Leukemia Related Osteolyses Detected by PET Successfully Treated with Cladribine and Ibandronate
}

\author{
Ena Rankovićc ${ }^{*}$, Antonija Balenovic ${ }^{2}$, Snjezana Dotlic ${ }^{3,4}$ and Igor Aurer ${ }^{1,4}$ \\ ${ }^{1}$ Division of Hematology, Department of Internal Medicine, University Hospital Centre Zagreb, Croatia \\ ${ }^{2}$ Department of Nuclear Medicine, Medical School, University of Rijeka and Health Care Center Zagreb, Croatia \\ ${ }^{3}$ Department of Pathology and Cytology, University Hospital Centre Zagreb, Croatia \\ ${ }^{4}$ School of Medicine, University of Zagreb, Croatia
}

*Corresponding author: Ranković E, Division of Hematology, Department of Internal Medicine, University Hospital Centre Zagreb, Kispaticeva 12, HR-1000 Zagreb, Croatia, Tel: +385-1-2388-644; E-mail: enarankovic@yahoo.com

Received: November 13, 2020; Accepted: November 21, 2020; Published: November 28, 2020

Keywords: Hairy cell leukemia; Osteolyses; Positrone-emission tomography; Cladribine; Diphosphonates

To the Editor,

Hairy cell leukemia (HCL) is a rare indolent lymphoproliferative disorder clinically characterized by pancytopenia and splenomegaly [1], with high overall survival rates after treatment with purine analogues [2-4]. We report a relatively young patient with HCL presenting with widespread osteolytic disease detected by PET who was successfully treated with cladribine and ibandronate. Osteolyses are not considered a frequent complication of HCL [5] and we are aware of only one case report describing the use of PET for diagnostic purposes in this setting [6]. Resolution of bone lesions has been described after treatment with radiotherapy or cladribine [6-9], but to our knowledge, there are no data on the use of bisphosphonates in this context.

A 45-year old man was referred for hematological evaluation of osteolyses. He had a history of long-standing right hip and new onset left hip pain with a gradual restriction of his sports activities. Hip MRI showed osteolytic changes in both femoral heads, while PET-CT showed multiple PET-avid osteolyses in the skeleton (FIG. 1). Physical examination and laboratory findings, including ESR, CBC, calcium, phosphorus, LDH, serum and urine protein electrophoresis and immunofixation, were normal. Since the trephine biopsy sample obtained from the right posterior superior iliac spine, an area not affected by osteolyses, was normal, a core-needle biopsy of the right femoral head was performed. The marrow was infiltrated with

Citation: Ranković E, Balenovic A, Dotlic S, et al. Hairy Cell Leukemia Related Osteolyses Detected by PET Successfully Treated with Cladribine and Ibandronate. Clin Case Rep Open Access. 2020;3(4):170. 
medium-sized villous lymphoid cells with abundant cytoplasm that were CD20+, CD25+, DBA44+, CD123+ and CD5, CD3, CD43 negative. HCL was diagnosed and the patient treated with a 5-day course of subcutaneous cladribine $0.14 \mathrm{mg} / \mathrm{kg} / \mathrm{day}$ and ibandronate $50 \mathrm{mg}$ daily with calcium and vitamin D supplementation. Platelets and hemoglobin levels remained normal, leukopenia was mild and transient. A PET-CT scan performed 4 months after the start of treatment showed FDG-negative osteolyses with bone regeneration (FIG. 1). Due to an area of increased uptake in the stomach upper endoscopy was performed and showed changes consistent with erosive, H. pylori negative gastritis, probably related to the use of biphosphonates. Ibandronate was stopped. A skeletal radiologic evaluation performed after 8 months showed remarkable normalization of bone structure. The patient resumed his high level of physical activities, and at the last follow up eight years after treatment, he was still in complete remission.

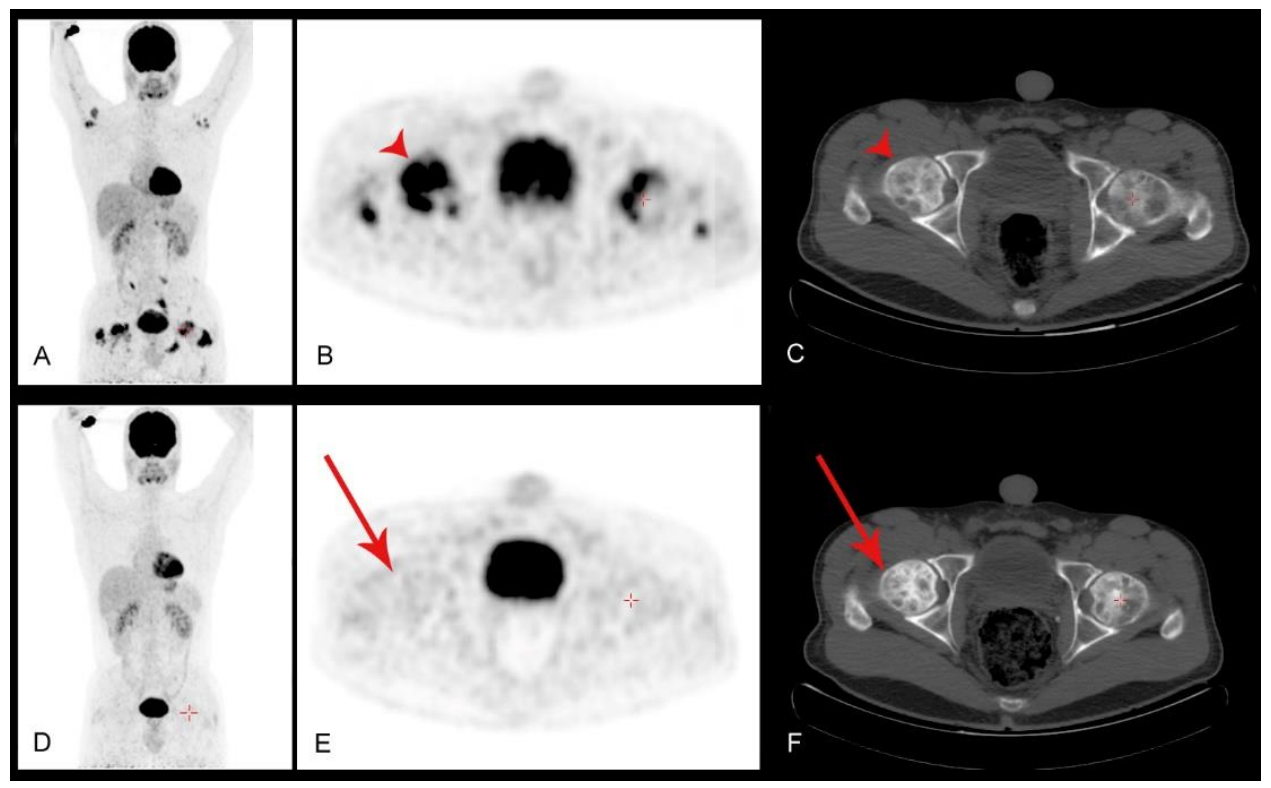

FIG. 1. (A) FDG-PET/CT at diagnosis showing osseous involvement in both arms, right scapula, L5 vertebrae, right iliac bone, left sacrum, both femoral heads and great trochanters, right acetabulum and left ischium corresponding to osteolytic lesions observed on CT. Transversal PET (B) and CT images (C) of hip regions with most intensive FDG activity (SUVmax= 7.6). Osteolytic lesions are indicated by arrow heads. (D, E) Follow-up study performed 4 months after the start of treatment showing complete clearance of metabolically active disease from all previously affected regions and partial sclerosation of both femoral heads (arrow) observed on a transversal CT image (F).

Our patient was unusual since his HCL presented initially with osteolyses without diffuse marrow involvement. This unusual tumor distribution is probably the reason why cladribine caused almost no reduction in blood counts. Since this was a young, physically active man with the affection of weight-bearing bones and extended skeletal changes which precluded radiotherapy, we decided to administer cladribine in combination with a highly active bisphosphonate. Our experience, suggests that osteolyses in HCL respond favorably to modern chemotherapy, possibly in combination with biphosphonates which highly effect bone regeneration, and that in cases with widespread skeletal changes, radiotherapy might be omitted. Another interesting aspect of this case was the use of PET. Our results, suggest that HCL-related osteolyses accumulate FDG and that PET can be used for evaluation of skeletal involvement in this disease. 


\section{Funding}

Supported in part by grant 108-1081872-1908 from the Croatian Ministry of Science.

\section{Acknowledgement}

The authors do not have any relevant conflicts of interest related to this publication.

\section{Ethical Standards}

Informed consent was obtained from the patient.

\section{REFERENCES}

1. Foucar K, Falini B, Stein H. Hairy cell leukaemia. In: Swerdlow SH, Campo E, Harris NL, et al., editors. WHO classification of tumours of haematopoietic and lymphoid tissues. Lyon: IARC, France; 2017. 226-8 p.

2. Goodman GR, Burian C, Koziol JA, et al. Extended follow-up of patients with hairy cell leukemia after treatment with cladribine. J Clin Oncol. 2003;21(15):891-6.

3. Dearden CE, Else M, Catovsky D. Long-term results for pentostatin and cladribine treatment of hairy cell leukemia. Leukemia Lymphoma. 2011;52 Suppl 2:21-24.

4. Aurer I, Mitrovic Z, Kovacevic-Metelko J, et al. Treatment of hairy cell leukemia with cladribine (in Croatian). Lijec Vjesn. 2007;129(3-4):80-3.

5. Filippi AR, Franco P, Marinone C, et al. Treatment options in skeletal localization of hairy cell leukemia: a systematic review on the role of radiation therapy. Am J Hematol. 2007;82(11):1017-21.

6. Rosen DS, Smith S, Gurbuxani S, et al. Extranodal hairy cell leukemia presenting in the lumbar spine. J Neurosurg Spine. 2008;9(4):374-6.

7. Lal A, Tallman MS, Soble MB, et al. Hairy cell leukemia presenting as localized skeletal involvement. Leuk Lymphoma. 2002; 43(11):2207-11.

8. Jehn U, Bartl R, Dietzfelbinger H, et al. An update: 12-years follow-up of patients with hairy cell leukemia following treatment with 2-chlorodeoxyadenosine. Leukemia. 2004;18(9):1476-81.

9. Gray MT, Rutherford MN, Bonin DM. Hairy-cell leukemia presenting as lytic bone lesions. J Clin Oncol. 2013; 31(25):e410-2. 